\title{
A new technique of percutaneously adjustable pulmonary artery banding
}

Shiv Kumar Choudhary, MCh, Sachin Talwar, MCh, Balram Airan, MCh, Raghunath Mohapatra, MS, Rajnish Juneja, DM, Shyam Sunder Kothari, DM, Anita Saxena, DM, and Panangipalli Venugopal, MCh

Objective: Pulmonary artery banding is associated with a high morbidity and mortality. We describe a new technique of adjustable pulmonary artery banding to prevent these problems.

Methods: Between December 2003 and May 2005, 32 patients aged 18 days to 2 years (mean age, $2.5 \pm 0.5$ months) and weighing 2.1 to $6.3 \mathrm{~kg}$ (mean, $3.6 \pm$ $1.3 \mathrm{~kg}$ ) underwent adjustable pulmonary artery banding.

Results: All patients survived the operation. There were 2 deaths, one caused by meningitis and another caused by aspiration pneumonitis. Satisfactory band gradients were achieved between 3 and 10 days $(7.2 \pm 2.6$ days $)$ in 3 to 6 sittings. Mean follow-up was $7.5 \pm 3.8$ months (1-16 months). One patient required reoperation for unsatisfactory band gradient 2 weeks after discharge. There were no late deaths. Follow-up computed tomographic angiograms $(\mathrm{n}=4)$ demonstrated proper band placement and ruled out distortion of the pulmonary arteries. Four patients underwent uneventful definitive operations after an interval of 7 to 13 months.

Conclusion: This technique of percutaneously adjustable pulmonary artery banding is simple and inexpensive and allows easy band adjustments without the need for multiple reoperations.

From the Cardiothoracic Centre, All India Institute of Medical Sciences, New Delhi, India.

This paper was selected as the best paper for "The Young Investigator Award" at the 7th Annual Conference of the Paediatric Cardiac Society of India, held at Hyderabad, India, July 16-18, 2005.

Received for publication Aug 23, 2005; revisions received Sept 29, 2005; accepted for publication Oct 10, 2005.

Address for reprints: Shiv Kumar Choudhary, MCh, Department of Cardiothoracic and Vascular Surgery, All India Institute of Medical Sciences, New Delhi, 110029, India (E-mail: shivchoudhary@ hotmail.com).

J Thorac Cardiovasc Surg 2006;131:621-4

0022-5223/\$32.00

Copyright $(9) 2006$ by The American Association for Thoracic Surgery

doi:10.1016/j.jtcvs.2005.10.037
$\mathrm{D}$ espite primary repair being the procedure of choice for most of the complex cardiac malformations, pulmonary artery banding (PAB) is still a useful initial palliation for a definite subset of patients. Since its inception, PAB has been accompanied with high mortality because of the sudden increase in the afterload to the ventricle. Difficulties of adjusting the appropriate band size also led to overbanding and underbanding, both contributing to higher morbidity and subsequent reoperations..$^{1-3}$ Various methods of adjustable PAB were proposed to eliminate these problems, ${ }^{3-8}$ but none of these methods became popular because of complexity and cost involvement. We have developed a simple, inexpensive, and effective method of adjustable $\mathrm{PAB}$, which is presented here.

\section{Methods}

Between December 2003 and May 2005, 2413 operations were performed for various congenital heart diseases. Of these, 32 patients aged 18 days to 2 years (mean age, $2.5 \pm 0.5$ months) and weighing 2.1 to $6.3 \mathrm{~kg}$ (mean, $3.6 \pm 1.3 \mathrm{~kg}$ ) underwent adjustable PAB at the All India Institute of Medical Sciences, New Delhi, India. The underlying cardiac diagnoses were multiple ventricular septal defects in isolation or with associated other system morbidities $(\mathrm{n}=15)$, univentricular heart $(\mathrm{n}=12)$, double-outlet right ventricle with additional anomalies $(\mathrm{n}=3)$, and atrioventricular canal defects $(\mathrm{n}=2)$.

Standard anesthesia techniques with endotracheal intubation were used. A small cannula was placed in the radial artery for arterial pressure monitoring, and 2 peripheral veins were cannulated for venous access. 


\section{Abbreviations and Acronyms \\ PA = pulmonary artery \\ $\mathrm{PAB}=$ pulmonary artery banding \\ $\mathrm{PTFE}=$ polytetrafluoroethylene}

Informed consent was obtained from all patients. The preferred approach was through a median sternotomy. Lately we have started performing this operation through an upper partial sternotomy in which only the manubrium is divided. After sternotomy, the thymus was split open but not excised. The pericardium was opened just enough to expose the great vessels. The aorta and the main pulmonary artery (PA) were separated from each other proximally. The ductus was dissected out and clipped in all cases. A right-angle forceps was passed between the aorta and the PA, and the PA was looped with a No. 2 Ethibond (Johnson and Johnson Inc, Somerville, NJ) suture. The right-angle forceps was again passed in a similar fashion, and the suture was grasped again by the tip of the right-angle forceps so that the PA was doubly looped. Now both the ends of the thread were passed through a $0.5-\mathrm{cm} \times 0.5-\mathrm{cm}$ polytetrafluoroethylene (PTFE) pledget (IMPRA, Inc, Tempe, Ariz), which was anchored to the adventitia of the PA with interrupted 6-0 polypropylene sutures to prevent migration of the band. Both the arms of the Ethibond suture were clipped to each other with a ligaclip (LT200; Ethicon EndoSurgery, Inc, Cincinnati, Ohio) flush with the PTFE pledget. These sutures were then brought out through the pericardium, which was closed after placing one temporary epicardial pacing wire on the right ventricle. After pericardial closure, the 2 ends of the suture were brought out through the lateral edge of the sternum and then through the subcutaneous tissue and skin. The sternum was then closed. The 2 ends of the suture were then passed through a 1-cm $\times 2$-cm PTFE pledget and clipped together with a big ligaclip (LT400, Ethicon Endo-Surgery, Inc). The ends of the suture were then tied together to form a loop. The patient was then shifted to the intensive care unit. The two ends of the suture were then tied together to form a loop (Figure 1).

Tightening of the band was started when possible after the patient was extubated and was on room air. If it was believed that the heart failure would prevent extubation without tightening of the band, it was carried out in increments enough to achieve extubation, and the final tightening was deferred. Tightening was achieved by placing additional clips outside between the PTFE pledget and the previous clip while the electrocardiogram and oxygen saturation with pulse oximetry were monitored. Echocardiographic assessment was performed simultaneously to estimate the band gradient and to assess the ventricular function. The tightening was performed in 3 to 6 sittings at intervals of 1 to 3 days, adding not more than $30 \mathrm{~mm} \mathrm{Hg}$ gradient at a time. In 2-ventricle candidates the target was to achieve a gradient of around $60 \mathrm{~mm} \mathrm{Hg}$ with a minimum acceptable saturation of $85 \%$.

For single-ventricle candidates, the band was tightened as much as possible. However, we ensured that the saturation did not decrease to less than $75 \%$. If there was hemodynamic compromise during tightening or there was bradycardia or a decrease in the saturation, the band was loosened by simply removing the lowermost clip and allowing the threads to retract down.
On achieving satisfactory gradients, the band was internalized by simply opening up the subcutaneous plane for about $1 \mathrm{~cm}$ and clipping the 2 threads together. Above this, the sutures were cut to release the portion of the band outside the skin. The skin was then closed with 2 interrupted sutures. We observed that the band gradient increased by 5 to $10 \mathrm{~mm} \mathrm{Hg}$ at the time of internalization.

After discharge from the hospital, these patients were followed up after 1 month and then at every 3-month interval. At each follow-up visit, they underwent clinical examination, measurement of saturation and echocardiography for band gradients, and estimation of PA pressure and ventricular function.

\section{Results}

All patients survived the operation. The median duration of mechanical ventilation was 6 hours (range, 2 hours to 20 days). No inotropes were required. There were 2 deaths, one caused by meningitis and another caused by aspiration pneumonitis. Satisfactory band gradients were achieved between 3 and 10 days ( $7.2 \pm 2.6$ days), and the band was internalized between 7 and 20 days (median, 10 days). Mean follow-up was $7.5 \pm 3.8$ months (1-16 months). One patient required reoperation for unsatisfactory band gradient 2 weeks after discharge. At the time of reoperation, the band was found to have become loose because of the slippage of clips anchoring the 2 threads in the subcutaneous plane. This was attributed to a technical error leading to improper band fixation.

There were no late deaths. Follow-up computed tomographic angiograms $(n=4)$ demonstrated proper band placement and ruled out distortion of the PAs. The band gradients increased by 2 to $12 \mathrm{~mm} \mathrm{Hg}$ without hemodynamic changes. Four patients underwent definitive operations after an interval of 7 to 13 months. In 2 patients undergoing a bidirectional Glenn shunt at reoperation, the main PA was divided and repaired with autologous glutaraldehyde-fixed pericardium. In the other 2 patients undergoing closure of ventricular septal defect, a longitudinal pulmonary arteriotomy was made across the band, and the PA was repaired with autologous glutaraldehyde-fixed pericardium. The remaining patients are awaiting reoperation.

\section{Discussion}

Conventional PAB has been reported to carry a high morbidity and mortality. ${ }^{1,2}$ A fixed band suddenly increases the afterload to the ventricle, and if the band is too tight, there can be bradycardia, hypotension, severe hypoxemia, and sometimes cardiac arrest. On the other hand, a loose band defeats the very purpose of this operation, and multiple reoperations might be required to achieve optimal gradients. There is no definitive guide to achieve satisfactory band tightening; intraoperative pressure measurements alone have not proved effective in evaluating the adequacy of the band. ${ }^{3-7}$ This problem is likely because the gradients are 
achieved when the patient is receiving a high concentration of inspired oxygen and is paralyzed and sedated.

For these critically ill patients, there is a need to develop a method of banding that is delicately adjustable not only during the operation but also postoperatively because the patient's hemodynamic and respiratory condition might change postoperatively, thereby altering the gradient created at the time of the operation. Several investigators have developed a variety of adjustable bands. ${ }^{3-8}$ Bonnet and colleagues ${ }^{8}$ have recently described their early results with a telemetric adjustable FloWatch pneumatic device in which tightening and loosening are regulated by an external control unit. Although effective, this device and the other described methods are expensive, require special equipment, and have not found general acceptance.

Gradual and delicate tightening and loosening of the band are possible with our method, making it particularly useful. Two-dimensional echocardiography and continuouswave Doppler study were used to ascertain the besttolerated degree of tightening along with the dynamic performance of right and left ventricular size and function. This is important because gradient alone is not an adequate means of assessing tolerable outflow obstruction. The most important advantage is that the tightening is carried out under physiologic conditions when the patient is off ventilator and off inotropes. Also, because the band is tightened at multiple sittings, the increase in afterload to the ventricle is gradual and can be adjusted to that tolerated by the patient without hemodynamic instability under normal physiologic conditions. It can be loosened immediately in the case of poor tolerance. Furthermore, this technique is simple, inexpensive, does not require specialized equipment, and is minimally invasive in those instances in which it is performed through a ministernotomy.

An initial concern about this type of band was that the PA could get tented toward the sternum and could lead to PA distortion, as well as making sternal re-entry hazardous at reoperation. To address these concerns, we performed computed tomographic angiography in 4 randomly selected patients at follow-up and found that these concerns were misplaced. Also, the sternal re-entry at reoperation was not difficult, and the PA was not adherent to the back of the sternum. Another concern was that the suture used for the band might cut through a dilated and tense PA. However, we have not observed this. Careful dissection and placement of the band, avoiding trauma to the adventitia of the PA, appear to have prevented this complication. Also, we initially feared pressure necrosis of the skin underlying the PTFE pledget; to prevent this, we ensured that the size of the pledget was large enough $(1 \mathrm{~cm} \times 2 \mathrm{~cm})$ to distribute the pressure in a wider area.

A limitation of our study is that it is an early experience, and we do not have direct measurements of the PA pressures. However, the echocardiographically achieved gradients

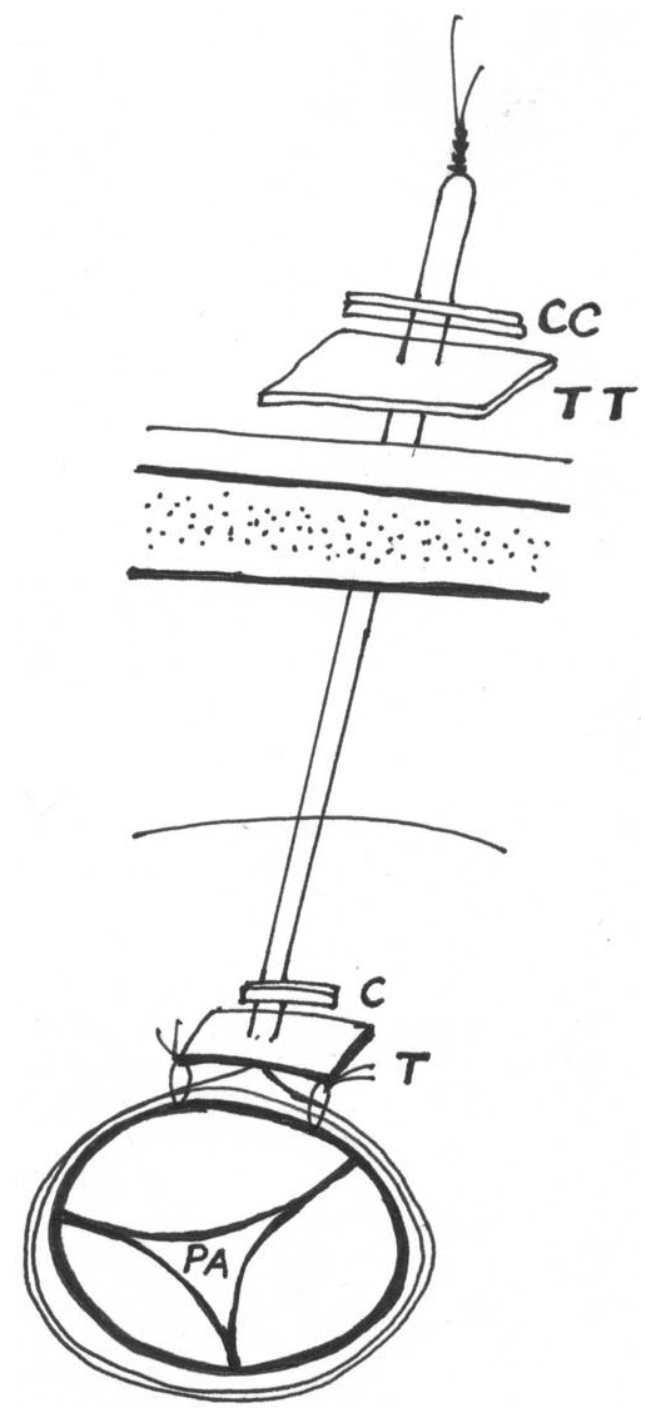

Figure 1. The technique of adjustable pulmonary artery banding. The Ethibond suture is doubly looped around the pulmonary artery $(P A)$, passed through a polytetrafluoroethylene (PTFE) pledget (T), and a clip $(C)$ is applied to both the threads flush with the pledget. They are then passed through the pericardium, sternum, subcutaneous tissue, and skin and through another PTFE pledget (TT), and another clip (CC) is applied above the TT before being tied together to form a loop. Subsequent tightening is done by placing additional clips between the outside clip (CC) and the TT.

have been satisfactory, and the band has been an adequate initial palliation in patients who have undergone reoperation. The findings at reoperation suggest that there were not many adhesions around the band, and there was no distortion of the PA. In addition, there was no band migration, and it was well positioned without compressing the left or right PAs.

None of our patients have required loosening of the band at follow-up. We believe that the purpose of the band is to 
provide short-term palliation, and any loosening that might be required in the initial postoperative phase can be easily achieved before internalization of the band, which is usually performed after 10 to 20 days. However, if long-term palliation is desired, the potential for somatic growth is a concern.

\section{Conclusions}

This technique of percutaneously adjustable PAB is simple, inexpensive, and allows easy band adjustments without the need for multiple reoperations.

We thank Dr H. V. Jayant Kumar for drawing Figure 1.

\section{References}

1. Stewart S, Harris P, Manning J. Pulmonary artery banding: an analysis of current risks, results and indication. $J$ Thorac Cardiovasc Surg. 1980;80:431-6.
2. Kirklin JW, Appelbaum A, Bargeron LM Jr. Primary repair versus banding for ventricular septal defects in infancy. In: Kidd BSL, Rowe $\mathrm{RD}$, editors. The child with congenital heart disease after surgery. Mt Kisco, NY: Futura; 1976. p 3-9.

3. Dajee H, Benson L, Laks H. An improved method of pulmonary artery banding. Ann Thorac Surg. 1984;37:254-7.

4. Le Bret E, Bonhoeffer P, Folliguet TA, Sidi D, Laborde F, de Leval MR, et al. A new percutaneously adjustable, thoracoscopically implantable, pulmonary artery banding: an experimental study. Ann Thorac Surg. 2001;72:1358-61.

5. Solis E, Heck CF, Seward JB, Kaye MP. Percutaneously adjustable pulmonary artery band. Ann Thorac Surg. 1986;41:65-9.

6. Warren ET, Heath BJ, Brand WW. A staged expanding pulmonary artery band. Ann Thorac Surg. 1992;54:240-3.

7. Vince DJ, Culham JAG. A prosthesis for banding of the main pulmonary artery, capable of serial dilatation by balloon angioplasty. $J$ Thorac Cardiovasc Surg. 1989;97:421-7.

8. Bonnet D, Corno AF, Sidi D, Sekarski N, Beghetti M, Schulze-Neick S, et al. Early clinical results of the telemetric adjustable pulmonary artery banding FloWatch-PAB. Circulation. 2004;110(suppl 1):II158-63.

\section{Availability of Journal back issues}

As a service to our subscribers, copies of back issues of The Journal of Thoracic and Cardiovascular Surgery for the preceding 5 years are maintained and are available for purchase from Elsevier Inc. until inventory is depleted. Please write to Elsevier Inc., Subscription Customer Service, 6277 Sea Harbor Dr, Orlando, FL 32877, or call 800-654-2452 or 407-345-4000 for information on availability of particular issues and prices. 